\title{
Ion current to a substrate in the pulsed DC hollow cathode plasma jet deposition system
}

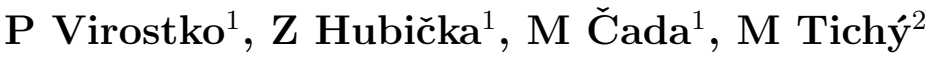 \\ ${ }^{1}$ Institute of Physics of the Academy of Sciences of the Czech Republic, v. v. i., \\ Na Slovance 2, 18221 Praha 8, Czech Republic \\ 2 Charles University in Prague, Faculty of Mathematics and Physics, \\ V Holešovičkách 2, 18000 Praha 8, Czech Republic \\ E-mail: milan.tichy@mff.cuni.cz
}

\begin{abstract}
The current of positive ions to a substrate was studied in the pulsed DC hollow cathode plasma jet system for deposition of thin films working at low pressures. Time evolution of electric current to a planar probe with pulsed negative DC bias was measured. The pulsing of the bias of the probe was applied to allow measurements with a dielectric film being deposited on its surface. The frequency of pulsing of the probe bias was higher than the frequency of pulsing of the discharge. Time evolution of the ion current density to the probe $j_{\mathrm{i}}$ was determined for the whole period of modulation of the discharge for the discharge repetition frequency $2.5 \mathrm{kHz}$ and different duty cycles $D$. The mean ion current density $\left\langle j_{\mathrm{i}}\right\rangle$, averaged over the whole period of the discharge, was rising with the decreasing $D$ although the value of the mean discharge current was kept constant. A simple theoretical model is proposed to explain this effect. It is shown that the observed rise of $\left\langle j_{\mathrm{i}}\right\rangle$ with decreasing $D$ is in major part caused by the rise of the ion current in the afterglow region of the pulsed discharge.
\end{abstract}

PACS numbers: 52.70.Ds, 52.77.-j

Submitted to: JPD 


\section{Introduction}

Pulsed excitation of discharges has been recently increasingly studied and employed in the plasma-assisted deposition of thin films [1,2]. Originally, pulsed excitation was proposed as a solution to problems connected with the undesired arcing during reactive sputtering. Moreover, it allows to apply much higher instantaneous power to the discharge than continuous excitation [3], leading to different conditions during sputtering and consequently to thin films with properties that might not be obtainable using continuous excitation.

Plasma diagnostic plays a key role in studying new methods and optimizing existing methods of deposition. Monitoring and control of plasma parameters during the deposition ensures namely the reproducibility of deposited films. One of the important parameters to study and observe is the current of positive ions from the plasma to a substrate. The ion bombardment of the substrate can significantly influence the parameters of deposited films, for example their surface morphology or structure $[4,5,6]$. The substrate is often held on a negative bias potential with respect to the bulk plasma to increase the energy of ions impinging on it and generally to control the ion bombardment.

The aim of the presented work is a study of the ion current to a negatively biased substrate in the pulsed direct current (DC) hollow cathode plasma jet system for deposition of thin films. The hollow cathode plasma jet (HCPJ) $[7,8,9]$ is a low-temperature sputtering device working at pressures approximately from $0.1 \mathrm{~Pa}$ to $100 \mathrm{~Pa}$. Its deposition rate is comparable to the usual deposition rate of magnetrons. It is favourable for depositions on substrates with complicated shapes or into holes because of the jet-like character of the discharge. It can also be used for sputtering of magnetic materials [10]. Pulse modulation of the power applied to the HCPJ allows to increase the power applied to the cathode, and consequently the sputtering of the cathode material, without overheating the cathode since the mean applied power including the space between pulses can be kept low.

The ion current was measured by a negatively biased planar probe immersed into the bulk plasma in the position of the substrate. The configuration of the HCPJ for deposition of $\mathrm{TiO}_{x}$ films was used. The titania films are dielectric and therefore the negative bias of the probe was periodically switched down to zero to discharge a positive charge that was accumulating on the surface of the film.

\section{Experimental setup}

A schematic representation of the experimental setup is in figure 1. The main part of the HCPJ is a cylindrical nozzle, which is made from the material to be sputtered. The nozzle is placed in a copper block, which is cooled by water. An insulating ceramic cover is placed around the copper block with the nozzle, see figure 1. More details about the HCPJ can be found in [9] and the works cited therein.

The nozzle is made from pure titanium in the HCPJ system for deposition of $\mathrm{TiO}_{x}$ 


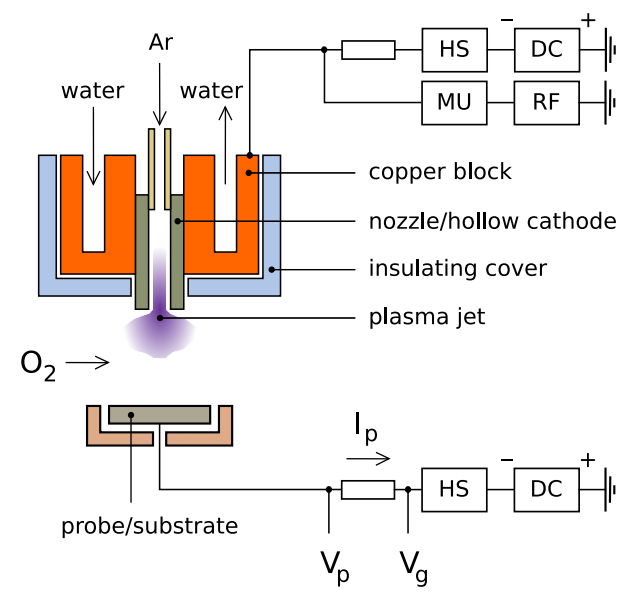

Figure 1. A schematic representation of the experimental setup. DC - DC generator, HS - high-voltage switch, MU - matching unit, RF - RF generator.

films, which was used in the experiments described in this work. The length of the nozzle was $30 \mathrm{~mm}$ and its internal diameter was $5 \mathrm{~mm}$. The outer diameter of the ceramic cover was $30 \mathrm{~mm}$.

The HCPJ was placed on the axis of a cylindrical stainless-steel vacuum chamber with the height of $35 \mathrm{~cm}$ and the diameter of $30 \mathrm{~cm}$. The outlet of the nozzle was in the distance approximately $10 \mathrm{~cm}$ from the flange, on which the whole system was mounted. The chamber was grounded.

Pure argon was fed through the nozzle into the chamber. A part of measurements was performed in pure argon and another part in the mixture of argon and molecular oxygen $\left(\mathrm{Ar} / \mathrm{O}_{2}\right)$. In the latter case the oxygen was fed sideways to the chamber to minimize the poisoning of the nozzle, which is acting as a target for sputtering. The chamber was continuously pumped by a turbomolecular pump backed by a Roots pump and a rotary oil pump. The attained ultimate pressure in the chamber was of the order of $10^{-7} \mathrm{~Pa}$. The working pressure was $2.7 \mathrm{~Pa}$ for the measurements in argon, with the flow rate set to $0.203 \mathrm{~Pa} \cdot \mathrm{m}^{-3} \cdot \mathrm{s}^{-1}(120 \mathrm{sccm})$, and $6.7 \mathrm{~Pa}$ for the measurements in $\mathrm{Ar} / \mathrm{O}_{2}$, with the flow rate of argon set to $0.203 \mathrm{~Pa} \cdot \mathrm{m}^{-3} \cdot \mathrm{s}^{-1}(120 \mathrm{sccm})$ and the flow rate of oxygen set to $0.101 \mathrm{~Pa} \cdot \mathrm{m}^{-3} \cdot \mathrm{s}^{-1}(60 \mathrm{sccm})$. The working pressure was measured by a capacitive gauge and the flow rates were set using mass flow controllers.

The copper block, which was in close contact with the nozzle, was connected to a negative output of a DC power generator through a resistor and a switch, see figure 1. The resistor was used to stabilize the discharge current. The high-voltage switch was used to pulse-modulate the output of the DC generator. A radio-frequency (RF) power generator was connected through a matching unit to a point between the resistor and the copper block. The RF generator was operated in a continuous mode and provided an auxiliary power to create a background $\mathrm{RF}$ discharge to stabilize the ignition of the DC discharge after the DC excitation was switched on. It was not possible to obtain a stable pulsed DC discharge without the auxiliary RF power with the experimental 
configuration we used. The RF generator was operated at frequency $13.56 \mathrm{MHz}$ and the auxiliary RF power applied to the HCPJ was approximately $3 \mathrm{~W}$.

The DC generator was periodically pulsed with the repetition frequency $2.5 \mathrm{kHz}$ and with the varied duty cycle set from 0.1 to 1 . A pulse-modulated DC glow discharge with the hollow cathode effect was ignited between the nozzle and the grounded walls of the vacuum chamber. The mean discharge current was always set to $500 \mathrm{~mA}$. When the duty cycle $D=1$ was used, the switch was continuously on and actually a DC HCPJ discharge with the discharge current $I_{\mathrm{d}}=500 \mathrm{~mA}$ was burning. Even in this case, the small auxiliary RF power was applied to the HCPJ for comparison with measurements at different duty cycles, although it was not necessary since the DC HCPJ discharge burns stably.

Time evolutions of the discharge current $I_{\mathrm{d}}$ and the corresponding nozzle voltage $V_{\mathrm{d}}$ are depicted in figure 2 for selected duty cycles. The time evolutions for discharge in

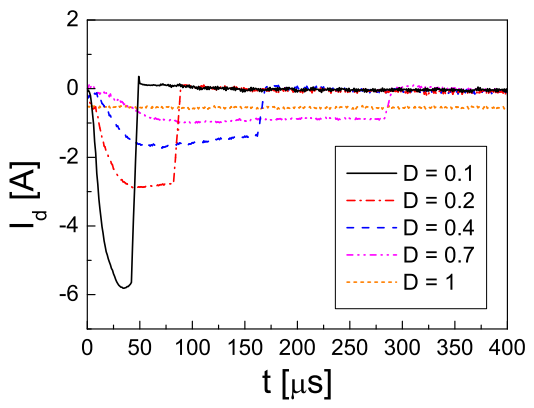

(a)

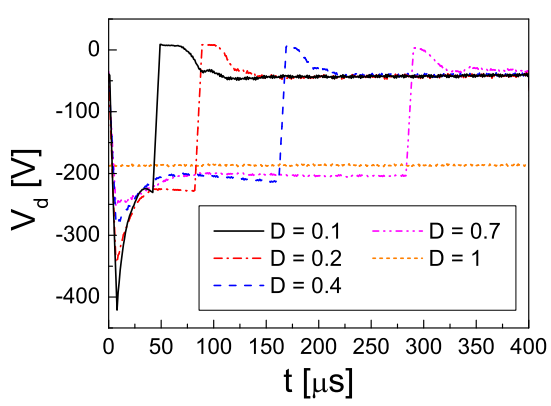

(b)

Figure 2. Time evolutions of the discharge current $I_{\mathrm{d}}$ and the voltage of the nozzle $V_{\mathrm{d}}$ for selected duty cycles $D$ and repetition frequency $2.5 \mathrm{kHz}$ of the pulsed $\mathrm{DC}$ HCPJ discharge in $\mathrm{Ar} / \mathrm{O}_{2}$. The mean discharge current was always $500 \mathrm{~mA} . D=1$ corresponds to the DC HCPJ with $I_{\mathrm{d}}=500 \mathrm{~mA}$.

$\mathrm{Ar} / \mathrm{O}_{2}$ are depicted; the time evolutions for discharge in argon were similar. The time evolutions depicted in figure 2 were obtained from the measured ones using adjacent averaging smoothing so that the RF component of the signals, corresponding to the auxiliary RF discharge, was removed. Consequently, the edges of the pulses in figure 2 were a bit distorted/slowed down; the original speeds of the falling edges of voltage $V_{\mathrm{d}}$, and the rising edges of both voltage $V_{\mathrm{d}}$ and current $I_{\mathrm{d}}$ were approximately $2 \mu \mathrm{s}$. The negative DC value of $V_{\mathrm{d}}$ during the space in between pulses corresponds to the DC self-bias of the nozzle due to the auxiliary RF discharge. The mean power applied to the nozzle is depicted in figure 3. The mean power was calculated as

$$
\left\langle P_{\mathrm{d}}\right\rangle=\frac{1}{T_{\mathrm{m}}} \int_{0}^{T_{\mathrm{m}}} I_{\mathrm{d}}(t) V_{\mathrm{d}}(t) \mathrm{d} t
$$

where $T_{\mathrm{m}}=400 \mu \mathrm{s}$ is the period of the discharge modulation.

The ion current was determined using a probe placed on the axis of the nozzle in the distance of $30 \mathrm{~mm}$ downstream from the nozzle outlet, i.e. in the usual position of 


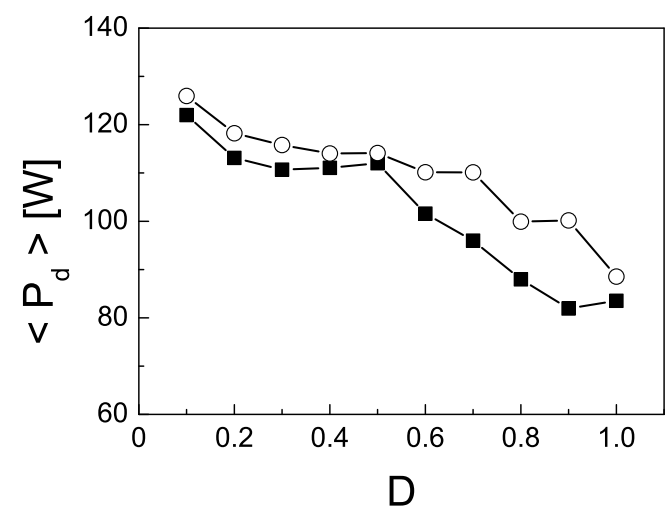

Figure 3. The mean power applied to the nozzle for different duty cycles.

- discharge in argon, $\mathrm{O}$ - discharge in $\mathrm{Ar} / \mathrm{O}_{2}$.

a substrate. The substrate holder, used for deposition experiments, was removed from the chamber. The probe was a planar copper electrode with the circular cross-section with the diameter $8.1 \mathrm{~mm}$. A ceramic cover was placed from beneath and around the electrode with the space between the electrode and the cover of approximately $1 \mathrm{~mm}$. The electrode was connected by a UHV-suitable coaxial cable to an electrical vacuum feedthrough. On the air side of the chamber a DC generator was connected to the feedthrough through a resistor and a high-voltage switch, see figure 1.

A pulsed negative DC voltage with the repetition frequency of $50 \mathrm{kHz}$ and the duty cycle approximately 0.5 was applied onto the probe. The time evolution of the electric current through the probe $I_{\mathrm{p}}$ was measured. Typical time evolution of $I_{\mathrm{p}}$ is depicted in figure 4 together with the voltage $V_{\mathrm{g}}$ measured between the switch and the resistor on the electrical line to the probe, see figure 1 . The generator of the discharge and the generator of the probe pulses were not synchronized. By taking the ion current samples from several consecutive discharge cycles it was possible to acquire the time dependence of the ion current almost continuously over the whole discharge period. The setpoint of voltage $V_{\mathrm{g}}$ during the pulses on the probe was held constant, see figure 4 . However, the voltage on the probe $V_{\mathrm{p}}$, see figure 1, was varying during the pulses on the probe because the voltage drop on the resistor between the switch and the probe was changing with the current $I_{\mathrm{p}}$, which was different at the different times after the start of the discharge pulse. The voltage $V_{\mathrm{g}}$ was set low enough to satisfy $V_{\mathrm{p}} \leq-40 \mathrm{~V}$ during the pulses on the probe for the whole period of pulse modulation of the discharge. The current $I_{\mathrm{p}}$ was approximately constant with $V_{\mathrm{p}}$ as was expected from the ion saturation current to a planar probe. Its value for $V_{\mathrm{p}}=-70 \mathrm{~V}$, which was the lowest voltage used, was only by about $6 \%$ higher than for $V_{\mathrm{p}}=-40 \mathrm{~V}$. The variaton of $I_{\mathrm{p}}$ with $V_{\mathrm{p}}$ was therefore neglected.

Depending on the instantaneous probe bias it is possible to follow in figure 4 the time dependence of the negative current (when the probe is grounded) as well as the positive current (when the probe is negatively biased) to the probe. It may seem strange that 


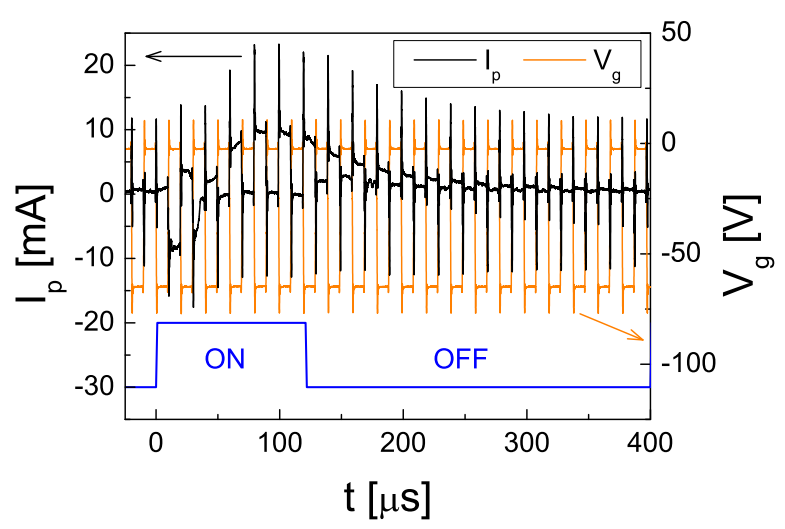

Figure 4. The time evolutions of the electric current through the probe $I_{\mathrm{p}}$ and the voltage $V_{\mathrm{g}}$ measured between the switch and the resistor on the electrical line to the probe. Pulsed DC HCPJ discharge in pure argon with $D=0.3$. The pulsing of the discharge is schematically indicated in the lower part of the graph.

the negative current component with the probe grounded is comparable to the positive ion current with the probe negatively biased. It is, however, necessary to stress that we cannot separate the positive ion and the electron current to the probe when the probe is grounded. While with the negatively biased probe we measure almost solely the positive ion current with grounded probe we measure the sum of the electron and positive ion current component. That is most probably due to the fact that the plasma potential at the position of the grounded probe lies somewhat above ground, i.e. the probe is sligthly negative with reference to plasma. The grounded probe therefore collects electrons as well as positive ions. The existence of slightly positive plasma potential in a DC driven hollow cathode plasma jet at a certain distance from the nozzle and similar experimental conditions was recently found in [12]. It was also found earlier [8] that the electron temperature in the active phase of the DC driven HCPJ is comparatively low. Hence the electron current to the grounded probe can be reduced down to the level of the ion current by even only small retarding voltage and the positive ion current component to the grounded probe cannot be neglected. Therefore we can see in figure 4 even by a naked eye that the probe positive current averaged over the discharge period exceeds the average value of the negative one. The data illustrated in figure 4 were taken in pure argon so that the only layer on the probe was the (conducting) layer of sputtered Ti.

Similar feature, i.e. that the probe positive current averaged over the discharge period exceeds somewhat the average value of the negative one, we observed also in experiments performed in mixture of argon and oxygen. We explain that by the fact that the experiments were made quite quickly and therefore the $\mathrm{TiO}_{x}$ layer, which was being deposited on the probe, did not continuously cover the whole surface of the probe and therefore it was still partly conducting. 


\section{Results and discussion}

The orientation of the probe current $I_{\mathrm{p}}$ was chosen as is depicted in figure 1 , from the probe to the generator. The current $I_{\mathrm{p}}$ is positive during the pulses of negative bias of the probe and equal to the current of positive ions $I_{\mathrm{i}}$ from the plasma to the probe since the probe voltage $V_{\mathrm{p}}$ is sufficiently negative to repel most electrons there. The composition of positive ions in the plasma under study has not been studied yet, but it can be estimated from the optical emission spectroscopy that the majority of positive ions are $\mathrm{Ar}^{+}$ions, and that some $\mathrm{Ti}^{+}$ions are present. As explained above the negative value of the current $I_{\mathrm{p}}$ during the time intervals when the probe bias is off means that electric current of electrons from the plasma to the probe is bigger than the electric current of positive ions there. Only the probe current $I_{\mathrm{p}}$ during the pulses of the probe bias, i.e. the total current of positive ions to a negatively biased probe $I_{\mathrm{i}}$, was studied further.

For one period of discharge modulation, the ion current $I_{\mathrm{i}}$ was obtained at equidistant time intervals, with spaces between the intervals corresponding to the times when the probe bias was off. Since the frequencies of the discharge and probe pulse generators were not synchronized the phase shift of the equidistant intervals of $I_{\mathrm{i}}$ with respect to the discharge modulation was arbitrary for each period of discharge modulation. The temporal dependence of the ion current $I_{\mathrm{i}}$ for the whole time interval $\left\langle 0, T_{\mathrm{m}}\right\rangle$, i.e. without the spaces due to the probe bias being off, was obtained by measuring the probe current $I_{\mathrm{p}}$ for several consecutive periods of the discharge modulation. Thanks to the arbitrary phase shift of probe bias modulation with respect to the discharge modulation, the intervals of the measured $I_{\mathrm{i}}$ covered the whole interval $\left\langle 0, T_{\mathrm{m}}\right\rangle$ typically in less than 10 consecutive discharge cycles.

The time evolution of the ion current density to the probe $j_{\mathrm{i}}$ was calculated by dividing the ion current $I_{\mathrm{i}}$ by the area of the probe. The measured time evolutions of $j_{\mathrm{i}}$ for different duty cycles of discharge modulation $D$ are depicted in figure 5 . The time evolution of $j_{\mathrm{i}}$ can be divided into the three parts: (i) a transient region after the discharge voltage pulse is turned on, (ii) a stable region during the pulse, and (iii) an afterglow region after the discharge voltage pulse is turned off.

There is a local maximum of $j_{\mathrm{i}}$ in the first region. This local maximum and in fact the whole time evolution of $j_{\mathrm{i}}$ corresponds to the time evolution of electron density $n_{\mathrm{e}}$ at the position of a substrate in the pulsed DC HCPJ, which was studied in [11]. In [11] it was suggested that the local maximum of $n_{\mathrm{e}}$ in the transient region can be caused by a short amplification of the auxiliary RF discharge. The hypothesis is following: the auxiliary RF discharge is immediately enhanced by application of high voltage to the nozzle at the start of the discharge pulse, while the hollow cathode discharge does not burn yet. This hypothesis is supported by the fact that the local maximum of $j_{\mathrm{i}}$ is bigger for shorter duty cycles, for which the negative voltage applied at the beginning of the discharge pulse to the nozzle has bigger magnitude, see figure 2(b). Then when the hollow cathode discharge starts, matching conditions change and the background 


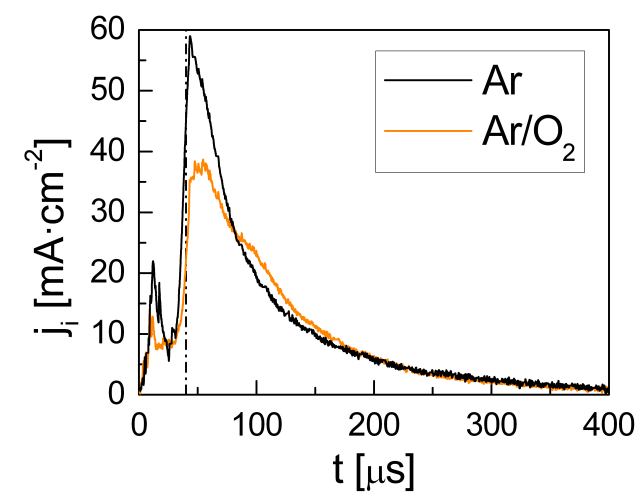

(a)

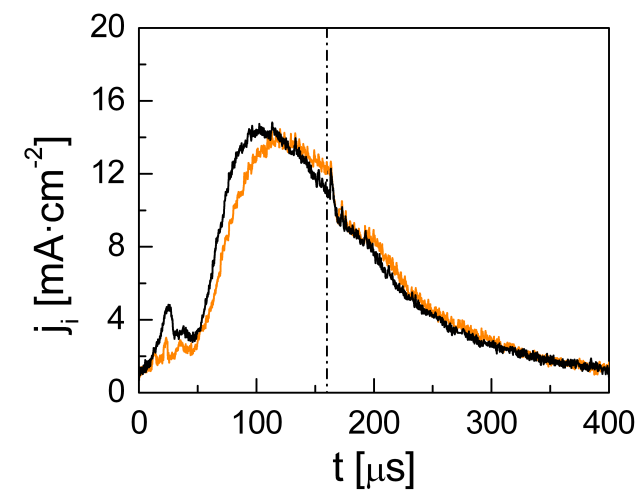

(c)

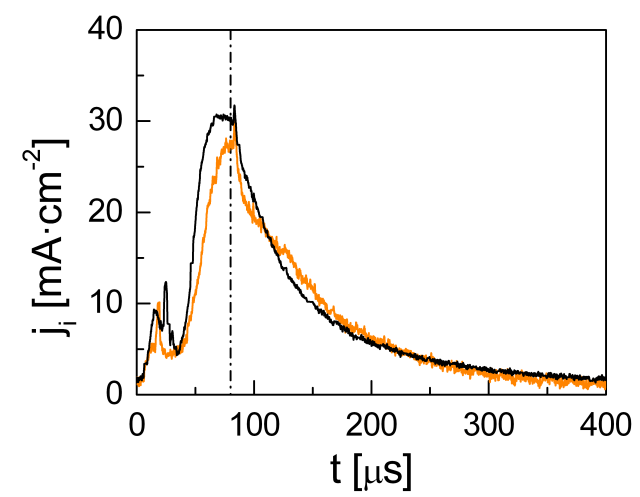

(b)

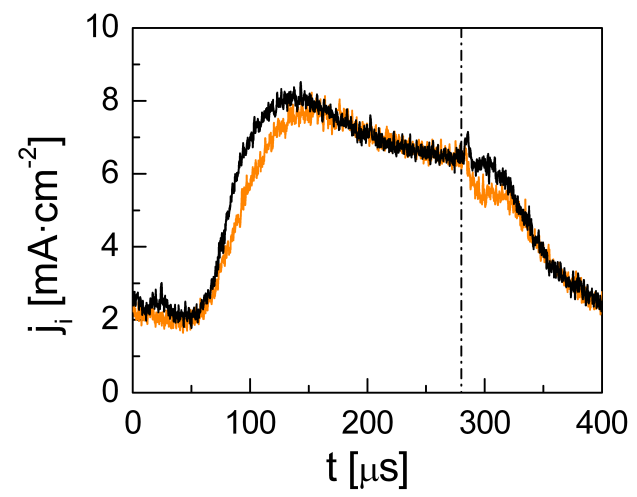

(d)

Figure 5. The time evolutions of the density of ion current to a substrate with the voltage $V_{\mathrm{p}} \leq-40 \mathrm{~V}$. The pulsed DC HCPJ discharge in argon and in $\mathrm{Ar} / \mathrm{O}_{2}$ with the mean discharge current $500 \mathrm{~mA}$, repetition frequency $2.5 \mathrm{kHz}$, and different duty cycle: (a) $D=0.1,(b) D=0.2,(c) D=0.4,(d) D=0.7$. The start of the pulse of discharge is at $t=0 \mathrm{~s}$ and the end of the pulse is indicated by a vertical dash-dot line in each graph. Note the different scale of the y-axis in different graphs.

RF discharge extinguishes (the RF-formed bias ceases).

The main rise of $j_{\mathrm{i}}$ starts approximately $50 \mu \mathrm{s}$ after the discharge voltage pulse is turned on. This delay is most probably caused by a finite velocity of propagation of the dense hollow-cathode plasma from the nozzle to the position of a substrate. From the known distance of the probe from the nozzle this velocity can be estimated to be $600 \mathrm{~m} \cdot \mathrm{s}^{-1}$. The propagation of the plasma from the nozzle can be also observed on the plasma parameters measured in [11] by a Langmuir probe at two different distances downstream from the nozzle, although the propagation was not discussed there. The velocity of the propagation estimated from the mentioned measurements in [11] is $800 \mathrm{~m} \cdot \mathrm{s}^{-1}$, i.e. similar to the velocity estimated form the delay of the rise of $j_{\mathrm{i}}$.

The ion current density $j_{\mathrm{i}}$ slightly decreases in the stable region during the pulse of the discharge. This is caused by a slightly decreasing discharge current during the pulse, see figure $2(a)$.

In the afterglow region, after the discharge pulse is turned off, $j_{\mathrm{i}}$ decreases 
approximately exponentially. This corresponds to the exponential decrease of $n_{\mathrm{e}}$ measured in [11], which means the plasma decays by diffusion to the grounded walls of the vacuum chamber.

It has to be noted that a fourth region was observed on the time evolution of plasma parameters in the pulsed DC HCPJ in [11]: a region of stable plasma parameters in the space in between pulses, with the plasma parameters corresponding to the auxiliary $\mathrm{RF}$ discharge. In this work, no similar region of stable $j_{\mathrm{i}}$ was observed in the space, due to the shorter space and lower auxiliary RF power applied here. The new pulse of discharge starts earlier than the plasma decays to a possible equilibrium state with the stable $j_{\mathrm{i}}$ corresponding to the auxiliary continuous RF discharge.

The presence of oxygen in the chamber did not significantly change the time evolution of $j_{i}$, most probably due to the fact that oxygen was added sideways and did not influence the processes in the hollow cathode too much.

The mean ion current density $\left\langle j_{\mathrm{i}}\right\rangle$ was determined by integration of the time evolution of $j_{\mathrm{i}}$ over the whole period of discharge modulation. The dependence of $\left\langle j_{\mathrm{i}}\right\rangle$ on the duty cycle of discharge modulation is depicted in figure 6. The mean ion current

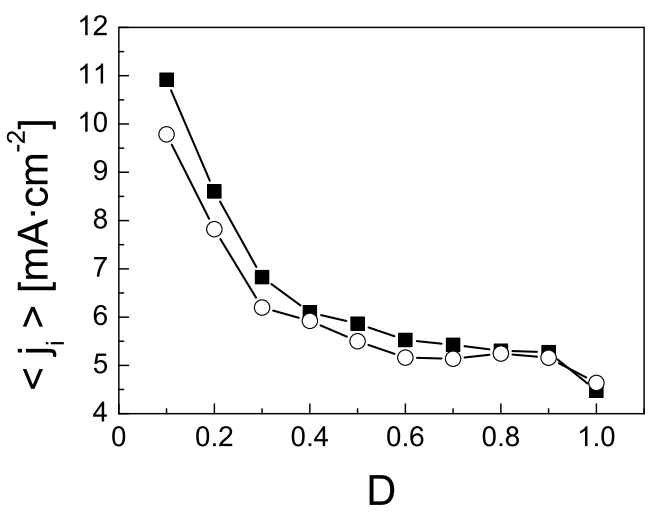

Figure 6. The dependence of the mean ion current density on the duty cycle of discharge modulation. - - discharge in argon, $\mathrm{O}$ - discharge in $\mathrm{Ar} / \mathrm{O}_{2}$.

to a substrate rises with the decreasing $D$ although the average discharge current is kept constant. Although the mean power applied to the discharge $\left\langle P_{\mathrm{d}}\right\rangle$ was rising with decreasing $D$, the relative rise of $\left\langle P_{\mathrm{d}}\right\rangle$ was much lower than the relative rise of $\left\langle j_{\mathrm{i}}\right\rangle$, especially for $D<0.4$, compare figure 3 and figure 6 . Therefore, the rise of $\left\langle j_{\mathrm{i}}\right\rangle$ cannot be explained by the rise of $\left\langle P_{\mathrm{d}}\right\rangle$ only.

We believe that the rise of $\left\langle j_{\mathrm{i}}\right\rangle$ with the decreasing $D$ can be qualitatively explained by a following theoretical model. In the model, $\left\langle j_{\mathrm{i}}\right\rangle$ is divided into the sum of two terms: one corresponding to the time interval of the pulse of the discharge and the second corresponding to the afterglow region in the space in between the pulses. The contribution of the auxiliary continuous RF discharge is neglected in the model. The 
division of $\left\langle j_{\mathrm{i}}\right\rangle$ into the two parts can be described as:

$$
\left\langle j_{\mathrm{i}}\right\rangle=\frac{1}{T_{\mathrm{m}}} \int_{0}^{T_{\mathrm{m}}} j_{\mathrm{i}}(t) \mathrm{d} t=\frac{1}{T_{\mathrm{m}}} \int_{0}^{T_{\mathrm{m}} D} j_{\mathrm{i}, \mathrm{p}}(t) \mathrm{d} t+\frac{1}{T_{\mathrm{m}}} \int_{T_{\mathrm{m}} D}^{T_{\mathrm{m}}} j_{\mathrm{i}, \mathrm{a}}(t) \mathrm{d} t
$$

where $j_{\mathrm{i}, \mathrm{p}}$ is the ion current density during the pulse and $j_{\mathrm{i}, \mathrm{a}}$ is the ion current density in the afterglow region.

The first term on the right-hand side of (2) is assumed to be proportional to the mean discharge current during the pulse. This assumption is based on the fact that $j_{\mathrm{i}}$ is directly proportional to the density of the charged particles that is in turn proportional to the discharge current in the HCPJ under the studied conditions [8]. The discharge current is further assumed to be zero in the afterglow region, which is well satisfied, see figure 2(a). Following these two assumptions, the first term on the right-hand side of (2) is proportional to the mean discharge current during the whole cycle, which was kept constant. Therefore, the mentioned term is constant as well, and will be marked $j_{\mathrm{i}, \mathrm{DC}}$ because it corresponds to the ion current density for a DC discharge with the same discharge current as is the mean discharge current in the pulsed DC discharge. It must be stressed that the assumption concerns only the mean ion current density during the pulse [the first term on the right-hand side of (2)] and no assumption about the actual time evolution of $j_{\mathrm{i}, \mathrm{p}}$ was made.

The time evolution of the ion current density in the afterglow region will be assumed in the form:

$$
j_{\mathrm{i}, \mathrm{a}}(t)=j_{\mathrm{i}, \mathrm{a} 0} \cdot \exp \left(-\frac{t-T_{\mathrm{m}} D}{\tau_{\mathrm{r}}}\right),
$$

which is the exponential decay from the value $j_{\mathrm{i}, \mathrm{a} 0}$ at the end of the discharge pulse, with $\tau_{\mathrm{r}}$ as the time constant of the decay.

The ion current density at the end of the discharge pulse is assumed to be:

$$
j_{\mathrm{i}, \mathrm{a} 0}=\frac{j_{\mathrm{i}, \mathrm{DC}}}{D} .
$$

At the equation (4) we arrived by considering the ideal case of a constant ion current density $j_{\mathrm{i}, \mathrm{p}}$ during the pulse. In such case, the current density $j_{\mathrm{i}, \mathrm{p}}$ equal to the righthand side of (4) satisfies the condition that the first term on the right-hand side of (2) is equal to $j_{\mathrm{i}, \mathrm{DC}}$.

In fact, the ion current density is not constant during the pulse, see figure 5 , but it can be expected that it satisfies (4) at the end of the pulse where $j_{\mathrm{i}}$ is stabilized, at least for higher $D$. Again, it has to be noted that no assumption is made regarding the actual time evolution of $j_{\mathrm{i}, \mathrm{p}}$, i.e. of the ion current density in the discharge pulse. Only the assumption (4) about the value of the ion current density at the end of discharge pulse is made. The ideal case of constant $j_{\mathrm{i}, \mathrm{p}}$ serves only to illustrate (4), and to explain how we arrived to that equation.

The proof that (4) is a good approximation is the figure 7 , in which the dependence of the experimentally determined product $j_{\mathrm{i}, \mathrm{a} 0} D$ on $D$ is depicted. The alignment of the data points in the figure 7 around the horizontal dash-dot lines that are defined by the values $j_{\mathrm{i}, \mathrm{DC}}$ determined for $D=1$ means that (4) can be in our case used to reasonably 


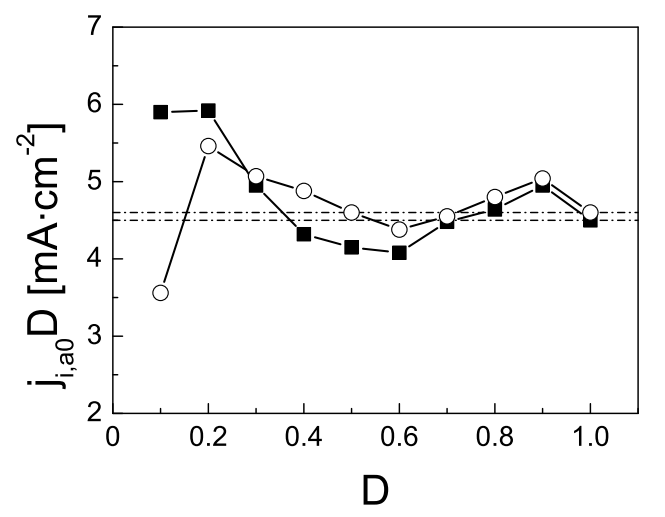

Figure 7. The product of the ion current density measured at the end of the pulse and the duty cycle for different duty cycles of pulsed DC excitation of the HCPJ discharge. - - discharge in argon, $\mathrm{O}$ - discharge in $\mathrm{Ar} / \mathrm{O}_{2}$. The horizontal dashdot lines indicate the values of $j_{\mathrm{i}, \mathrm{DC}}$ determined for the DC discharge (rightmost points, $D=1)$.

Table 1. The parameters to determine $\left\langle j_{\mathrm{i}}\right\rangle$ according to the model equation (5) for the pulsed DC HCPJ with the repetition frequency of the discharge $2.5 \mathrm{kHz}$ and the mean discharge current $500 \mathrm{~mA}$.

\begin{tabular}{llll}
\hline & $j_{\mathrm{i}, \mathrm{DC}}\left[\mathrm{mA} \cdot \mathrm{cm}^{-1}\right]$ & $\tau_{\mathrm{r}}[\mu \mathrm{s}]$ & $T_{\mathrm{m}}[\mu \mathrm{s}]$ \\
\hline $\mathrm{Ar}$ & 4.5 & 80 & 400 \\
$\mathrm{Ar} / \mathrm{O}_{2}$ & 4.6 & 90 & 400 \\
\hline
\end{tabular}

determine $j_{\mathrm{i}, \mathrm{a} 0}$ from a single parameter $j_{\mathrm{i}, \mathrm{DC}}$ for different $D$, with a good agreement with experiment for $D \geq 0.3$, for which duty cycles the ion current density is already stabilized at the end of the discharge pulse.

The mean ion current density can be determined from (2)-(4) together with the assumptions given above as:

$$
\left\langle j_{\mathrm{i}}\right\rangle=j_{\mathrm{i}, \mathrm{DC}}+\frac{j_{\mathrm{i}, \mathrm{DC}}}{D} \frac{\tau_{\mathrm{r}}}{T_{\mathrm{m}}}\left[1-\exp \left(-\frac{T_{\mathrm{m}}-T_{\mathrm{m}} D}{\tau_{\mathrm{r}}}\right)\right] .
$$

This equation is the theoretical model of $\left\langle j_{\mathrm{i}}\right\rangle$, in which $D$ is the independent variable and $j_{\mathrm{i}, \mathrm{DC}}, \tau_{\mathrm{r}}$, and $T_{\mathrm{m}}$ are the parameters.

The values of the parameters are listed in table 1 . The period of pulse modulation of the discharge $T_{\mathrm{m}}$ was fixed. The ion current density $j_{\mathrm{i}, \mathrm{DC}}$ was determined experimentally for a DC discharge with the discharge current $500 \mathrm{~mA}$ under the same conditions, including the auxiliary RF power applied. The time constant $\tau_{\mathrm{r}}$ was for a particular working gas determined as the average value of the time constants determined for each $D$ from the decrease of $j_{\mathrm{i}}$ in the afterglow region. This decrease is not exactly exponential in the whole afterglow region and for all $D$, but it can be fitted well linearly in the semilogarithmic scale, i.e. according to (3), between $50 \mu$ s and $100 \mu$ s after the pulse is turned off for all cases. The time constants $\tau_{\mathrm{r}}$ in table 1 were determined from the fits 
in this time interval.

The comparison of the model with the experimentally determined dependence of $\left\langle j_{\mathrm{i}}\right\rangle$ on $D$ is in figure 8 . It can be seen that the model is qualitatively in a very good

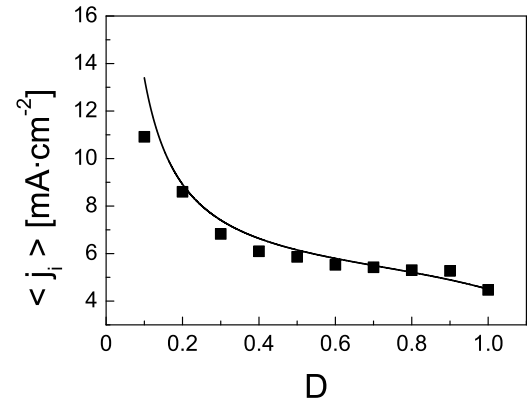

(a)

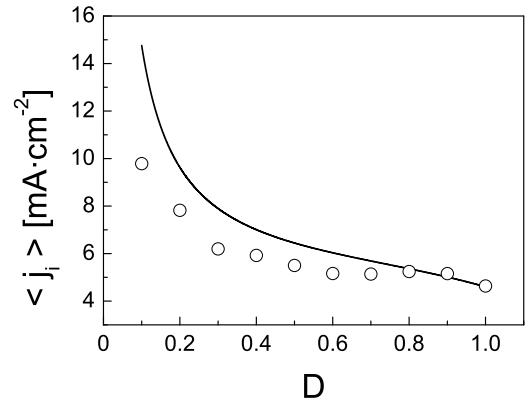

(b)

Figure 8. The comparison of the model with the experimentally determined dependence of the mean ion current density to a substrate on the duty cycle of the discharge modulation: line - model, symbols - experiment. (a) Discharge in Ar, (b) discharge in $\mathrm{Ar} / \mathrm{O}_{2}$.

agreement with the experimentally determined data - the model curve has a very similar shape to the experimentally determined dependence, including the steep rise of $\left\langle j_{\mathrm{i}}\right\rangle$ for $D<0.4$. A very good quantitative agreement between the model and the experiment for the discharge in argon, see figure $8(a)$, is more likely just a coincidence in view of the rough assumptions of the model.

From the agreement of the model with the data it can be concluded that the model equation (5) describes well the behaviour of $\left\langle j_{\mathrm{i}}\right\rangle$ with the changing duty cycle $D$ under the presented discharge conditions. According to the model, the rise of $\left\langle j_{\mathrm{i}}\right\rangle$ with the decreasing $D$ is caused by the rising contribution of the second term on the right-hand side of (2), i.e. by the rising contribution of the ion current in the afterglow region. That can be qualitatively explained, since for lower $D$ the decay of the ion current in the afterglow region starts from a higher value, see (4), and lasts longer (due to a longer idle time in between pulses while $\tau_{\mathrm{r}}$ does not differ significantly with $D$ ) than for higher $D$, thus contributing more to the mean ion current. The contribution of the ion current in the afterglow part of the discharge cycle to the mean ion current is then more significant for lower duty cycles.

\section{Conclusion}

The current of positive ions to a substrate was studied in the pulsed DC HCPJ system for depositions of thin films. The time evolution of the density of the ion current $j_{\mathrm{i}}$ was measured for the discharge repetition frequency of $2.5 \mathrm{kHz}$ and different duty cycles $D$. Measurements in argon and in the mixture of argon with molecular oxygen, used for actual deposition experiments of $\mathrm{TiO}_{x}$, were performed. 
The time evolution of $j_{\mathrm{i}}$ corresponds to the time evolution of electron density $n_{\mathrm{e}}$ at the position of a substrate in the pulsed DC HCPJ. A local maximum of $j_{\mathrm{i}}$ is present in the transition region after the pulse of the discharge is turned on, which can possibly be caused by a short amplification of the auxiliary RF discharge by the appearance of the high negative voltage on the nozzle. The main rise of $j_{\mathrm{i}}$ is delayed by approximately $50 \mu \mathrm{s}$ after the pulse is turned on, which is most probably caused by a finite velocity of propagation of the dense plasma from the nozzle to the position of a substrate.

The mean ion current density (ion density averaged over the discharge modulation period) $\left\langle j_{\mathrm{i}}\right\rangle$ was determined. The mean ion current density is rising with the decreasing $D$ although the mean discharge current is kept constant. The rise of the mean ion current density cannot be wholly explained by a relatively smaller rise of the mean power applied to the discharge that was observed for decreasing $D$. To qualitatively explain the increase of $\left\langle j_{\mathrm{i}}\right\rangle$ a simple theoretical model was presented. In the model, $\left\langle j_{\mathrm{i}}\right\rangle$ is divided into the sum of two terms: (i) one accounting for the contribution of $\left\langle j_{\mathrm{i}}\right\rangle$ during the discharge pulse and (ii) the other accounting for the contribution of $\left\langle j_{\mathrm{i}}\right\rangle$ during the afterglow part of the period, i.e. in the space in between discharge pulses.

The model corresponds well with the experimentally determined dependence of $\left\langle j_{\mathrm{i}}\right\rangle$ on $D$. According to the model, the rise of $\left\langle j_{\mathrm{i}}\right\rangle$ with the decreasing $D$ is caused by the significantly rising contribution of the ion current in the afterglow region with the decreasing $D$. We can then conclude that the use of a pulsed mode of the discharge increases the mean ion current to a substrate, especially at low duty cycles, in the deposition experiments of $\mathrm{TiO}_{x}$ with the DC HCPJ system with mean discharge current kept constant.

\section{Acknowledgments}

This work was supported by projects KAN301370701 and M100100915 of the Academy of Sciences of the Czech Republic, by projects 202/09/0800 and 202/09/P159 of the Grant Agency of the Czech Republic, and by project MSM0021620834 of the Ministry of Education, Youth, and Sports of the Czech Republic.

\section{References}

[1] Vlček J, Pajdarová A D and Musil J 2004 Contrib. Plasm. Phys. 44 426-36

[2] Bradley J W and Welzel T 2009 J. Phys. D: Appl. Phys. 42093001

[3] Helmersson U, Lattermann M, Bohlmark J, Ehiasarian A P, and Gudmundsson J T 2006 Thin Solid Films 513 1-24

[4] Musil J and Kadlec S 1990 Vacuum 40 435-44

[5] Phinichka N, Chandra R and Barber Z H 2004 J. Vac. Sci. Technol. A 22 477-81

[6] Olaya J J, Wei G, Rodil S E, Muhl S and Bhushan B 2007 Vacuum 81 610-8

[7] Bárdoš L and Berg S 1992 Surf. Coat. Tech. 54-55 91-5

[8] Virostko P, Hubička Z, Adámek P, Čada M, Olejníček J, Tichý M and Š́cha M 2006 Contrib. Plasm. Phys. 46 445-50 
[9] Hubička Z 2007 Low Temperature Plasmas. Fundamentals, Technologies, and Techniques vol 2 ed R Hippler et al (Weinheim: WILEY-VCH) pp 715-37

[10] Kraus L, Chayka O, Touš J, Fendrych F, Pirota K R, Šícha M and Jastrabík L 2001 J. Magn. Magn. Mater. 226-230 669-70

[11] Tichý M et al 2009 Plasma Sources Sci. Technol. 18014009

[12] Leshkov S, Kudrna P, Chichina M, Klusoň J, Picková I, Hubička Z, Tichý M, Contrib. Plasma Phys., submitted. 